Research Article

\title{
Kinematics Analysis of 6-DoF Articulated Robot with Spherical Wrist
}

\author{
Seemal Asif $(\mathbb{D}$ and Philip Webb \\ School of Aerospace, Transport and Manufacturing, Cranfield University, Bedford MK43 OAL, Cranfield, UK \\ Correspondence should be addressed to Seemal Asif; s.asif@cranfield.ac.uk
}

Received 29 November 2020; Revised 30 December 2020; Accepted 18 January 2021; Published 2 February 2021

Academic Editor: Fateh Mebarek-Oudina

Copyright (C) 2021 Seemal Asif and Philip Webb. This is an open access article distributed under the Creative Commons Attribution License, which permits unrestricted use, distribution, and reproduction in any medium, provided the original work is properly cited.

\begin{abstract}
The aim of the paper is to study the kinematics of the manipulator. The articulated robot with a spherical wrist has been used for this purpose. The Comau NM45 Manipulator has been chosen for the kinematic model study. The manipulator contains six revolution joints. Pieper's approach has been employed to study the kinematics (inverse) of the robot manipulator. Using this approach, the inverse kinematic problem is divided into two small less complex problems. This reduces the time of analysing the manipulator kinematically. The forward and inverse kinematics has been performed, and mathematical solutions are detailed based on D-H (Denavit-Hartenberg) parameters. The kinematics solution has been verified by solving the manipulator's motion. It has been observed that the model is accurate as the motion trajectory was smoothly followed by the manipulator.
\end{abstract}

\section{Introduction}

Locomotion is the process of causing a rigid body to move. The body needs force to move. Dynamics is the study of the motion of the body in which forces are modelled which helps the body to move, whereas kinematics is the geometrical study of the motion of the body without considering the forces that can affect the motion of the body.

Kinematics is the motion description of the rigid body. [1] Links are the connectivity body/member between joints. The kinematic chain is a grouping of links connected by joints, as illustrated in Figure 1. In the kinematic chain, the number of DoF (degree of freedom) is equal to the number of joints.

Maintaining a strong connection between the two joints is called the kinematics function of a link. This connection can be described with the following factors:

(i) $a$ : link length

(ii) $\alpha$ : link twist

Link length is measured along the line which is mutually perpendicular to both joints/axes. The perpendicularity in joints always exists except when both joints are parallel. Link twist is the angle of projection from the previous joint $(i-1)$ to the next joint (i) onto the axis $i-1$ (previous joint); the projection line is parallel to the next joint (axis i). The relationship between link length and twist is described in Figure 2 .

(i) A joint axis is formed at the connection of two links. This joint will have two parameters (one for each link) connected to it. These parameters are as follows:

(ii) $d$ : distance between links

(iii) $\theta$ : angle between links

The relative position or distance between the links is called link offset. Figure 3 describes these parameters, in which the joint angle is the angle between the links.

The four parameters demonstrated above are associated with each link. Axes can be aligned using these parameters. The parameters are also known as Denavit-Hartenberg link parameters. These are illustrated in Table 1 below:

The link numbering convention follows from the base of the arm till the last moving link. As mentioned in Figure 4, the first link is the connection between the base and first joint. 


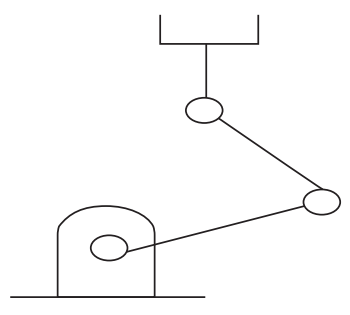

(a)

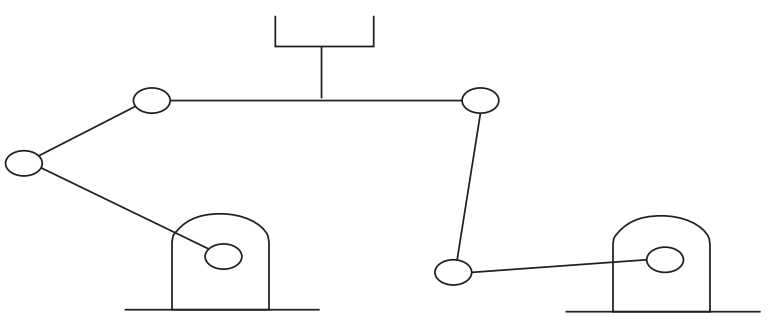

(b)

Figure 1: Kinematics of the manipulator: (a) open chain; (b) closed chain [2].

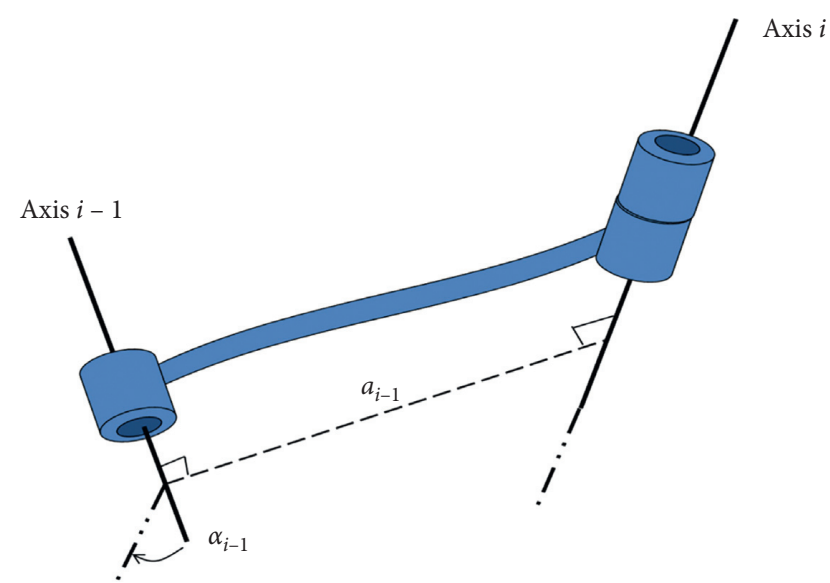

FIGURE 2: Kinematic function of link.

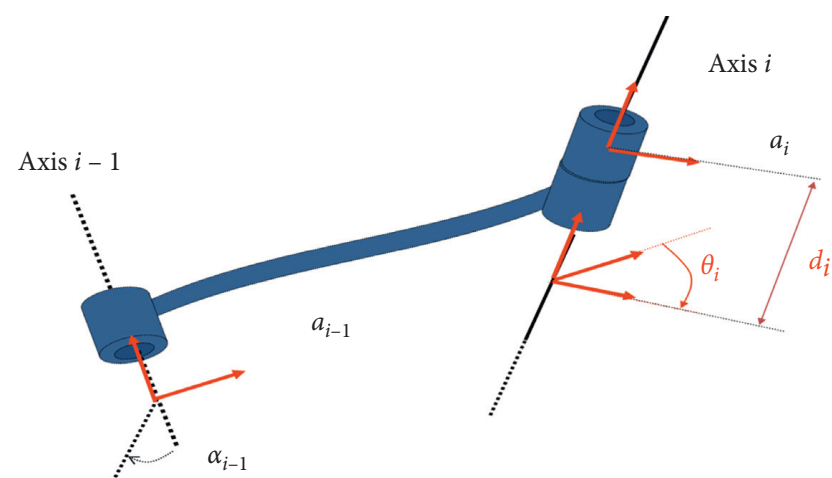

FIGURE 3: Link and joint parameters. Joint parameters are highlighted in red.

TABLe 1: Denavit-Hartenberg link parameters.

\begin{tabular}{lc}
\hline Link parameters & Joint parameters \\
\hline$a:$ link length & $d$ : distance between links \\
$\alpha$ : link twist & $\theta:$ angle between links \\
\hline
\end{tabular}

The parameters mentioned above in Table 1 are used for kinematic modelling of the robot. In kinematics, modelling the geometry of the robot is represented. Homogenous transformation (of the matrix) is commonly used as the definition of the kinematics model (particularly for chains mechanism). As described below,

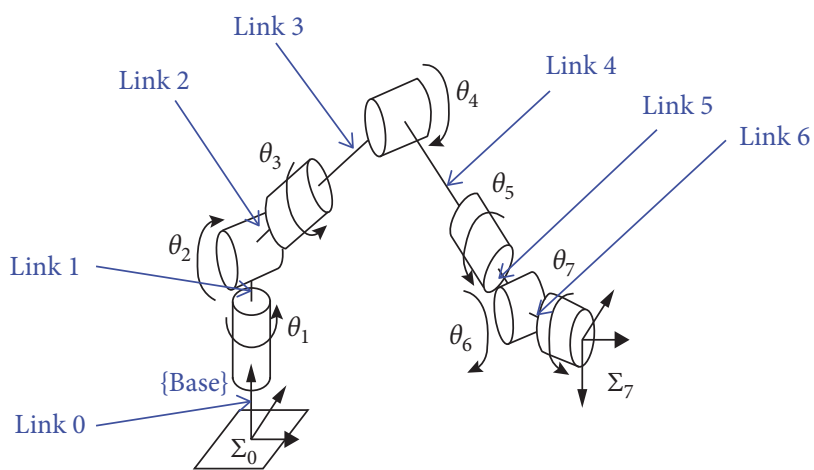

Figure 4: A 7-DoF manipulator arm [3].

$$
{ }_{n}^{0} T=T_{1} T_{2} T_{3} \ldots T_{i} \ldots T_{n}
$$

where $n$ is the number of links, $T_{i}$ is the link transformation from the $i^{\text {th }}$ joint, and ${ }_{n}^{0} T$ is the final pose for end-effector relative to the base.

There are two main types of kinematic models: forward kinematics and inverse kinematics. In forward kinematics, the length of each link and angle of each joint is given, and through that, position of any point $(x, y, z)$ can be found. In inverse kinematics, the length of each link and position of some points $(x, y, z)$ is given, and the angle of each joint is needed to find to obtain that position.

Several models are developed for kinematic modelling, but the D-H (Denavit-Hartenberg) model [4] is the most popular model. Limitations of the $\mathrm{D}-\mathrm{H}$ model are discussed, and CPC (completeness and parametric continuity) model and its mapping with the $\mathrm{D}-\mathrm{H}$ model were proposed [5]. The parametric continuity of the CPC model was achieved by using singularity free line representation.

\section{Forward Kinematics of Comau NM45}

The Comau NM45 [6] is a medium-scale robot. It has 6 degree of freedom joints. It is an articulated arm with a spherical wrist. The wrist joint intersects at one point. Figure 5 shows the manipulator with its link length and working envelope.

Forward kinematics is the study of the manipulator to find out its tip or end-effector position and orientation by using joint values of the manipulator. The first step of performing the forward kinematics is to label link lengths. 


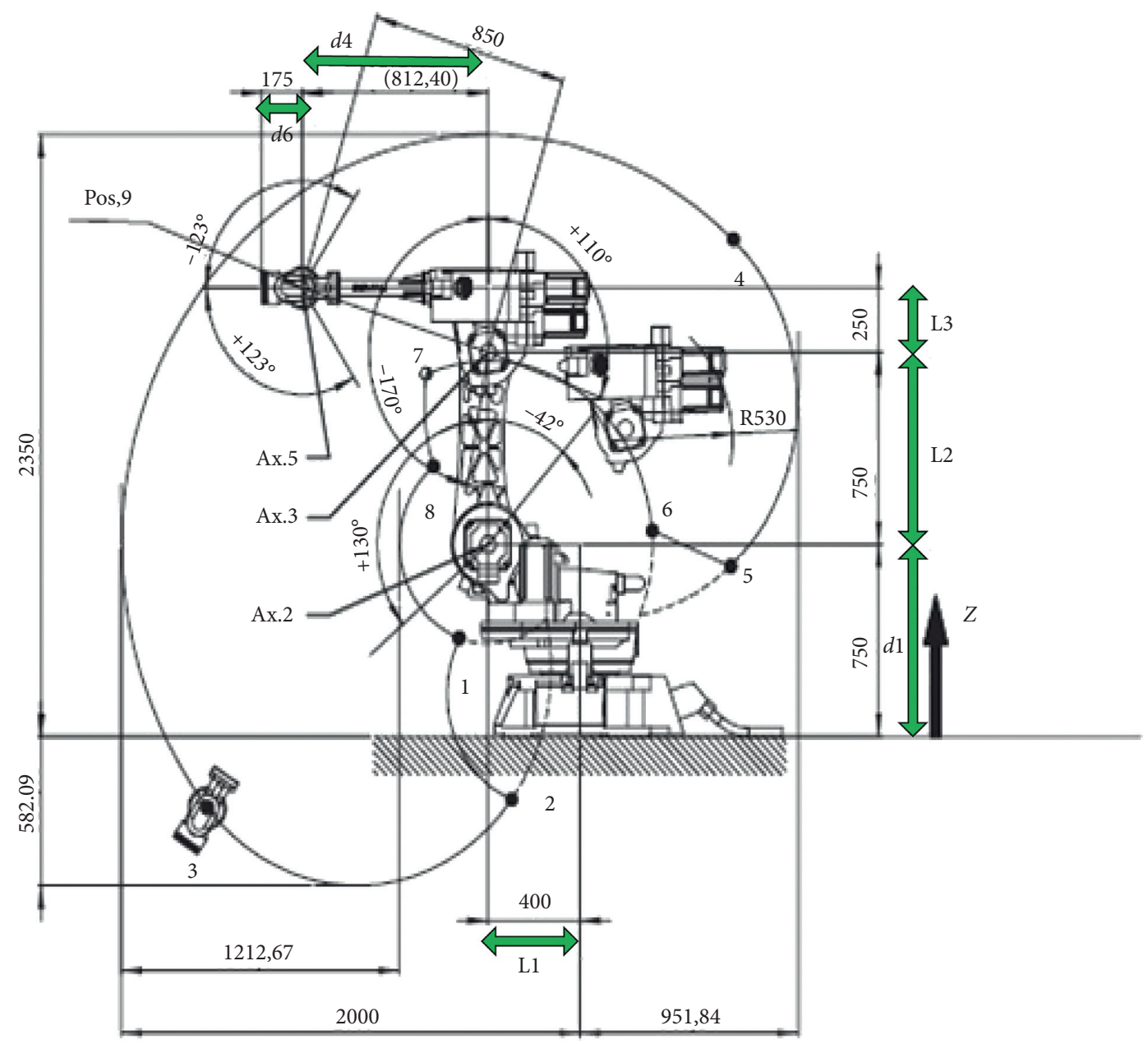

Figure 5: Comau NM45 (inline) operative area and link lengths.

This step has been performed in Figure 5. The second step to find the forward kinematics of the manipulator is to assign the frames. The frame assignment is done in Figure 6.

The D-H parameters can be found based on the frame assignment. The modified DH convention has been used for the frame assignment and $\mathrm{DH}$ parameters [7, 8]. These parameters are illustrated in Table 2.

The transformation matrix for a link $\mathbf{i}$ is described as follows:

$$
A_{i}=\left[\begin{array}{cccc}
\cos \theta i & -\sin \theta i \cos \alpha i & \sin \theta i \sin \alpha i & \alpha i \cos \theta i \\
\sin \theta i & \cos \theta i \cos \alpha i & -\cos \theta i \sin \alpha i & \alpha i \sin \theta i \\
0 & \sin \alpha i & \cos \alpha i & \mathrm{~d} i \\
0 & 0 & 0 & 1
\end{array}\right] .
$$

$A_{1}$ is the transformation matrix $T_{1}^{0}$ :

$$
T_{1}^{0}=\left[\begin{array}{cccc}
\cos \theta 1 & -\sin \theta 1 \cos \alpha 1 & \sin \theta 1 \sin \alpha 1 & \alpha 1 \cos \theta 1 \\
\sin \theta 1 & \cos \theta 1 \cos \alpha 1 & -\cos \theta 1 \sin \alpha 1 & \alpha 1 \sin \theta 1 \\
0 & \sin \alpha 1 & \cos \alpha 1 & d 1 \\
0 & 0 & 0 & 1
\end{array}\right] \text {, }
$$

$$
T_{1}^{0}=\left[\begin{array}{cccc}
\cos \theta 1 & 0 & \sin \theta 1 & \alpha 1 \cos \theta 1 \\
\sin \theta 1 & 0 & -\cos \theta 1 & \alpha 1 \sin \theta 1 \\
0 & 1 & 0 & d 1 \\
0 & 0 & 0 & 1
\end{array}\right]
$$

whereas $a 1=l 1=0.4$ and $d 1=0.75$; by placing these values above, the following equation which is the resultant for the transformation between the base and joint 1 is obtained:

$$
T_{1}^{0}=\left[\begin{array}{cccc}
\cos \theta 1 & 0 & \sin \theta 1 & \alpha 1 \cos \theta 1 \\
\sin \theta 1 & 0 & -\cos \theta 1 & \alpha 1 \sin \theta 1 \\
0 & 1 & 0 & 0.75 \\
0 & 0 & 0 & 1
\end{array}\right]
$$

For $\mathrm{A} 2=T_{2}^{1}$,

$$
T_{2}^{1}=\left[\begin{array}{cccc}
\cos \theta 2 & -\sin \theta 2 & 0 & \alpha 2 \cos \theta 2 \\
\sin \theta 2 & \cos \theta 2 & 0 & \alpha 2 \sin \theta 2 \\
0 & 0 & 1 & d 2 \\
0 & 0 & 0 & 1
\end{array}\right],
$$

and $\alpha=90$, so $T_{1}^{0}$ will be as follows: 


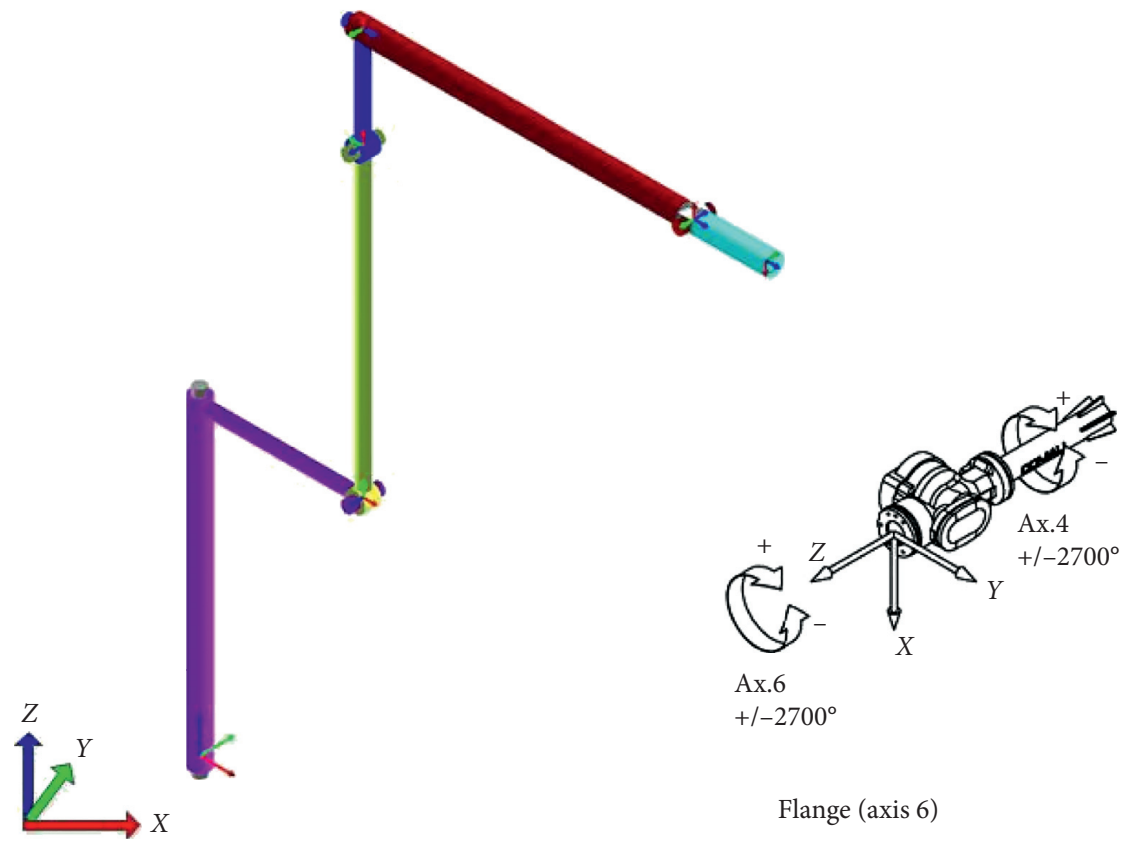

Figure 6: NM45 model with coordinates mapping.

TABle 2: Comau NM45 DH parameters.

\begin{tabular}{lcccc}
\hline Link & Link length $(\alpha i), \mathrm{m}$ & Twist angle $(\alpha i)$, deg & Joint offset & Joint angle $(\theta i)$, deg \\
\hline 1 & $L 1=0.4$ & 90 & $D 1=0.75$ & $(\theta 1)$ \\
2 & $L 2=0.75$ & 0 & 0 & $(\theta 2)$ \\
3 & $L 3=0.25$ & 90 & 0 & $(\theta 3)$ \\
4 & 0 & -90 & $D 4=0.8124$ & $(\theta 4)$ \\
5 & 0 & 90 & 0 & $(\theta 5)$ \\
6 & 0 & 0 & $D 6=0.175$ & $(\theta 6)$ \\
\hline
\end{tabular}

whereas $a 2=l 2=0.75$ and $d 2=0$; by placing these values above, the following equation which is the resultant for the transformation between joint 1 and joint 2 is obtained:

$$
T_{2}^{1}=\left[\begin{array}{cccc}
\cos \theta 2 & -\sin \theta 2 & 0 & 0.75 \cos \theta 2 \\
\sin \theta 2 & \cos \theta 2 & 0 & 0.75 \sin \theta 2 \\
0 & 0 & 1 & 0 \\
0 & 0 & 0 & 1
\end{array}\right] .
$$

For $\mathrm{A} 3=T_{3}^{2}$,

$$
T_{3}^{2}=\left[\begin{array}{cccc}
\cos \theta 3 & 0 & \sin \theta 3 & \alpha 3 \cos \theta 3 \\
\sin \theta 3 & 0 & -\cos \theta 3 & \alpha 3 \sin \theta 3 \\
0 & 1 & 0 & d 3 \\
0 & 0 & 0 & 1
\end{array}\right],
$$

whereas $a 3=l 3=0.25$ and $d 3=0$; by placing these values above, the following equation which is the resultant for the transformation between joint 2 and joint 3 is obtained:

$$
T_{3}^{2}=\left[\begin{array}{ccccc}
\cos \theta 3 & 0 & \sin \theta 3 & 0.25 \cos \theta 3 \\
\sin \theta 3 & 0 & -\cos \theta 3 & 0.25 \sin \theta 3 \\
0 & 1 & 0 & 0 \\
0 & 0 & 0 & 1
\end{array}\right] .
$$

For $\mathrm{A} 4=T_{4}^{3}$ and $\alpha=90$,

$$
T_{4}^{3}=\left[\begin{array}{cccc}
\cos \theta 4 & 0 & -\sin \theta 4 & \alpha 4 \cos \theta 4 \\
\sin \theta 4 & 0 & \cos \theta 4 & \alpha 4 \sin \theta 4 \\
0 & -1 & 0 & d 4 \\
0 & 0 & 0 & 1
\end{array}\right],
$$

whereas $a 4=0$; by placing the value of $a 4$ in the above equation, the following equation which is the resultant for the transformation between joint 3 and joint 4 is obtained:

$$
T_{4}^{3}=\left[\begin{array}{cccc}
\cos \theta 4 & 0 & -\sin \theta 4 & 0 \\
\sin \theta 4 & 0 & \cos \theta 4 & 0 \\
0 & -1 & 0 & d 4 \\
0 & 0 & 0 & 1
\end{array}\right] .
$$

For $\mathrm{A} 4=T_{5}^{4}$,

$$
T_{5}^{4}=\left[\begin{array}{cccc}
\cos \theta 5 & 0 & \sin \theta 5 & \alpha 5 \cos \theta 5 \\
\sin \theta 5 & 0 & -\cos \theta 5 & \alpha 5 \sin \theta 5 \\
0 & 1 & 0 & d 5 \\
0 & 0 & 0 & 1
\end{array}\right],
$$


whereas $a 5=0$ and $d 5=0$; by placing these values in the above equation, the following equation which is the resultant for the transformation between joint 4 and joint 5 is obtained:

$$
T_{5}^{4}=\left[\begin{array}{cccc}
\cos \theta 5 & 0 & \sin \theta 5 & 0 \\
\sin \theta 5 & 0 & -\cos \theta 5 & 0 \\
0 & 1 & 0 & 0 \\
0 & 0 & 0 & 1
\end{array}\right]
$$

For $\mathrm{A} 5=\mathrm{T}_{6}^{5}$ and $\alpha=0$,

$$
T_{6}^{5}=\left[\begin{array}{cccc}
\cos \theta 6 & -\sin \theta 6 & 0 & \alpha 6 \\
\sin \theta 6 & \cos \theta 6 & 0 & \alpha 6 \sin \theta 6 \\
0 & 0 & 1 & d 6 \\
0 & 0 & 0 & 1
\end{array}\right],
$$

whereas $a 6=0$; by placing the value of $a 6$ in the above equation, the following equation which is the resultant for the transformation between joint 5 and joint 6 is obtained:

$$
T_{6}^{5}=\left[\begin{array}{cccc}
\cos \theta 6 & -\sin \theta 6 & 0 & 0 \\
\sin \theta 6 & \cos \theta 6 & 0 & 0 \\
0 & 0 & 1 & d 6 \\
0 & 0 & 0 & 1
\end{array}\right]
$$

2.1. Transformation. For simplification, the following has been substituted:

$$
\begin{aligned}
& \theta 1=u, \\
& \theta 2=v, \\
& \theta 3=w, \\
& \theta 4=a, \\
& \theta 5=b, \\
& \theta 6=m .
\end{aligned}
$$

For $A 12=T_{2}^{0}=T_{1}^{0} \times T_{2}^{1}$, substitute values from equations (5) and (7):

$$
T_{2}^{0}=T_{1}^{0} X T_{2}^{1}=\left(\begin{array}{cccc}
\cos (u) & 0 & \sin (u) & 0.4 \cos (u) \\
\sin (u) & 0 & -\cos (u) & 0.4 \sin (u) \\
0 & 1 & 0 & 0.75 \\
0 & 0 & 0 & 1
\end{array}\right) \cdot\left(\begin{array}{cccc}
\cos (v) & -\sin (v) & 0 & 0.75 \cos (v) \\
\sin (v) & \cos (v) & 0 & 0.75 \sin (v) \\
0 & 0 & 1 & 0 \\
0 & 0 & 0 & 1
\end{array}\right)
$$

The resultant transformation between the base and joint 2 is illustrated as follows:

$$
T_{2}^{0}=\left[\begin{array}{cccc}
\cos (u) \cos (v) & -\cos (u) \sin (v) & \sin (u) & \cos (u)(0.75 \cos (v)+0.4) \\
\cos (v) \sin (u) & -\sin (u) \sin (v) & -\cos (u) & (0.75 \cos (v)+0.4) \sin (u) \\
\sin (v) & \cos (v) & 0 & 0.75 \sin (v)+0.75 \\
0 & 0 & 0 & 1
\end{array}\right],
$$

For A123 $=T_{3}^{0}=T_{1}^{0} \times T_{2}^{1} \times T_{3}^{2}$, for simplicity,

$$
\begin{aligned}
\mathrm{m} & =\cos (u)(0.75 \cos (v)+0.4), \\
n & =(0.75 \cos (v)+0.4) \sin (u), \\
u & =0.75 \sin (v)+0.75 \\
T_{3}^{0} & =T_{2}^{0} X T_{3}^{2}=\left(\begin{array}{cccc}
\cos (u) \cos (v) & -\cos (u) \sin (v) & \sin (u) & m \\
\cos (v) \sin (u) & -\sin (u) \sin (v) & -\cos (u) & n \\
\sin (v) & \cos (v) & 0 & o \\
0 & 0 & 0 & 1
\end{array}\right) \cdot\left(\begin{array}{cccc}
\cos (w) & 0 & \sin (w) & 0.25 \cos (w) \\
\sin (w) & 0 & -\cos (w) & 0.25 \sin (w) \\
0 & 1 & 0 & 0 \\
0 & 0 & 0 & 1
\end{array}\right) .
\end{aligned}
$$


The resultant transformation between the base and joint 3 is illustrated as follows:

$$
T_{3}^{0}=\left(\begin{array}{cccc}
\cos (u) \cos (v+w) & \sin (u) & \cos (u) \sin (v+w) & m+0.25 \cos (u) \cos (v+w) \\
\cos (v+w) \sin (u) & -\cos (u) & \sin (u) \sin (v+w) & n+0.25 \cos (v+w) \sin (u) \\
\sin (v+w) & 0 & -\cos (v+w) & 0+0.25 \sin (v+w) \\
0 & 0 & 0 & 1
\end{array}\right)
$$

For $\mathrm{A} 456=T_{6}^{3}=T_{4}^{3} \times T_{5}^{4} \times T_{6}^{5}$,

$$
\begin{aligned}
& A 45=T_{5}^{3}=T_{4}^{3} \times T_{5}^{4}=\left(\begin{array}{cccc}
\cos (a) & 0 & -\sin (a) & 0 \\
\sin (a) & 0 & \cos (a) & 0 \\
0 & -1 & 0 & 0.8124 \\
0 & 0 & 0 & 1
\end{array}\right) \cdot\left(\begin{array}{cccc}
\cos (b) & 0 & \sin (b) & 0 \\
\sin (b) & 0 & -\cos (b) & 0 \\
0 & 1 & 0 & 0 \\
0 & 0 & 0 & 1
\end{array}\right) \text {, } \\
& A 45=T_{5}^{3}=\left(\begin{array}{cccc}
\cos (a) \cos (b) & -\sin (a) & \cos (a) \sin (b) & 0 \\
\sin (a) \cos (b) & \cos (a) & \sin (a) \sin (b) & 0 \\
-\sin (b) & 0 & \cos (b) & 0.8124 \\
0 & 0 & 0 & 1
\end{array}\right) \\
& T_{6}^{3}=T_{4}^{3} \times T_{5}^{4} \times T_{6}^{5}=T_{5}^{3} \times T_{6}^{5}=\left(\begin{array}{cccc}
\cos (a) \cos (b) & -\sin (a) & \cos (a) \sin (b) & 0 \\
\sin (a) \cos (b) & \cos (a) & \sin (a) \sin (b) & 0 \\
-\sin (b) & 0 & \cos (b) & d^{4} \\
0 & 0 & 0 & 1
\end{array}\right) \cdot\left(\begin{array}{cccc}
\cos (m) & -\sin (m) & 0 & 0 \\
\sin (m) & \cos (m) & 0 & 0 \\
0 & 0 & 1 & d^{6} \\
0 & 0 & 0 & 1
\end{array}\right) .
\end{aligned}
$$

The resultant transformation between joint 3 and joint 6 is illustrated as follows:

$$
T_{6}^{3}=\left(\begin{array}{cccc}
\cos (a) \cos (b) \cos (m)-\sin (a) \sin (m) & -\cos (m) \sin (a)-\cos (a) \cos (b) \sin (m) & \cos (a) \sin (b) & d^{6} \cos (a) \sin (b) \\
\cos (b) \cos (m) \sin (a)+\cos (a) \sin (m) & \cos (a) \cos (m)-\cos (b) \sin (a) \sin (m) & \sin (a) \sin (b) & d^{6} \sin (a) \sin (b) \\
-\cos (m) \sin (b) & \sin (b) \sin (m) & \cos (b) & \cos (b) d^{6}+d^{4} \\
0 & 0 & 0 & 1
\end{array}\right) .
$$

Spherical wrist position can be extracted using the last column of $T_{6}^{0}$ transformation matrix, where

$$
T_{6}^{0}=\left[\begin{array}{cccc}
r_{11} & r_{12} & r_{13} & P_{x} \\
r_{21} & r_{22} & r_{23} & P_{y} \\
r_{31} & r_{32} & r_{33} & P_{z} \\
0 & 0 & 0 & 1
\end{array}\right],
$$

Which means the $T_{6}^{0}$ the transformation matrix can be represented in terms of $R_{6}^{0}$ and $P_{6}^{0}$ illustrated as follows:

$$
T_{6}^{0}=\left[\begin{array}{cc}
R_{6}^{0} & P_{6}^{0} \\
0 & 1
\end{array}\right],
$$

and spherical wrist position is illustrated as

$$
P_{6}^{0}=\left[\begin{array}{c}
P x \\
P y \\
P z
\end{array}\right] \text {. }
$$

Transformation matrix from base to end-effector is 


$$
\begin{aligned}
T_{6}^{0} & =T_{1}^{0} \times T_{2}^{1} \times T_{3}^{2} \times T_{4}^{3} \times T_{5}^{4} \times T_{6}^{5}, \\
T_{6}^{0} & =\left[\begin{array}{cccc}
r 11 & r 12 & r 13 & P x \\
r 21 & r 22 & r 23 & P y \\
r 31 & r 32 & r 33 & P z \\
0 & 0 & 0 & 1
\end{array}\right], \\
T_{6}^{0} & =\left[\begin{array}{cc}
R_{6}^{0} & P_{6}^{0} \\
0 & 1
\end{array}\right] .
\end{aligned}
$$

\section{Inverse Kinematics of Comau NM45}

Inverse kinematics is finding the joint values $(\theta 1, \theta 2, \theta 3, \theta 4, \theta 5, \theta 6)$ of the robot arm for the given position $(p)$ and orientation $(o)$. For inverse kinematics, the inverse orientation $R$ and inverse position $P$ are needed.

The Comau NM45 is an articulated arm with a spherical wrist. For finding anthropomorphic/articulated arm position and joint values $\theta 1, \theta 2, \theta 3$, the inverse position is needed.

Let $O_{c}$ be intersecting the last 3 joints. The motion of joints 4,5 , and 6 will not change the position of $O_{c}$, as stated in Figure 7 [19] This is according to Pieper's approach [7], in which the manipulator is divided to analyse the inverse kinematics [10].

According to $T_{6}^{3}$ matrix from equation (22), the position $P_{6}^{3}$ is always as mentioned in the following equation:

$$
P_{6}^{3}=\left[\begin{array}{c}
0 \\
0 \\
d_{\partial 6}
\end{array}\right] .
$$

So, the position $O$ will be as follows:

$$
O=O_{c}^{0}+d_{6} R\left[\begin{array}{l}
0 \\
0 \\
1
\end{array}\right],
$$

where $O$ is the position $P_{6}^{0}$ and $\mathrm{R}$ is the orientation which is $R$. The above equation can be written in terms of $O_{c}^{0}$ as follows:

$$
O_{c}^{0}=O-d_{6} R\left[\begin{array}{l}
0 \\
0 \\
1
\end{array}\right],
$$

The first three joints can be found in the following steps. They will determine the position of the manipulator:

$$
\begin{aligned}
O & =\left[\begin{array}{l}
o_{x} \\
o_{y} \\
o_{z}
\end{array}\right], \\
O_{c}^{0} & =\left[\begin{array}{l}
x_{c} \\
y_{c} \\
z_{c}
\end{array}\right], \\
{\left[\begin{array}{l}
x_{c} \\
y_{c} \\
z_{c}
\end{array}\right] } & =\left[\begin{array}{l}
o_{x}-d_{6} r_{13} \\
o_{x}-d_{6} r_{23} \\
o_{x}-d_{6} r_{33}
\end{array}\right] .
\end{aligned}
$$

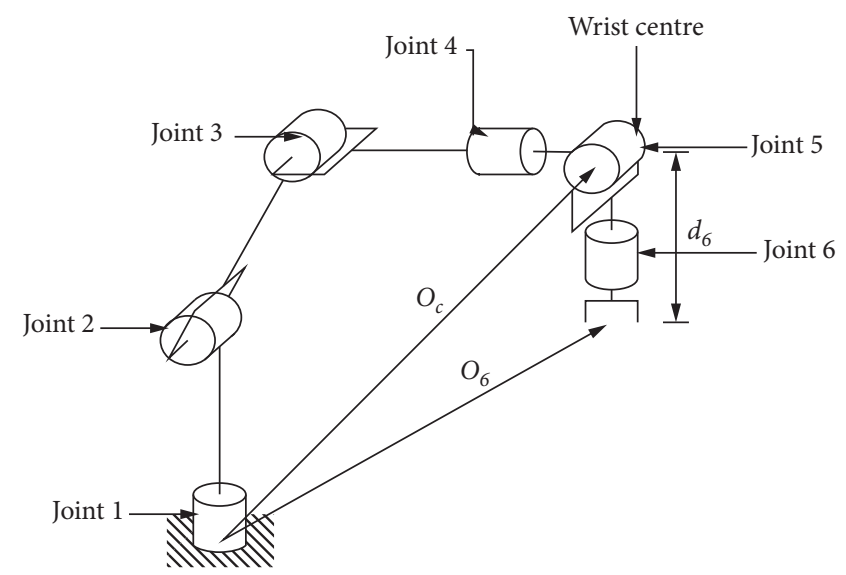

FIgURE 7: Kinematics decoupling (Spong, Hutchinson, and Vidyasagar, 2006).

For the orientation, the last three joints orientation is needed. The following equation shows the overall rotation of the manipulator in terms of $R_{3}^{0}$ and $R_{6}^{3}$ :

$$
R=R_{3}^{0} R_{6}^{3} .
$$

Rearrangement of equation (31) will yield $R_{6}^{3}$ below, through which the last three joint angles can be found:

$$
R_{6}^{3}=R_{3}^{0-1} R \longrightarrow \theta 4, \theta 5, \theta 6 .
$$

For articulated manipulator, the first three joints tell the position, as illustrated in Figure 8.

Projection of wrist onto $x_{0}, y_{0}$ plane has been shown in Figure 9.

This projection yields the triangle through which the angle value for $\theta 1$ can be found as follows:

$$
\theta 1=\mathrm{A} \tan 2\left(x_{c}, y_{c}\right) .
$$

If the wrist is rotated, then it will result in the following equation:

$$
\theta 1=A \tan 2\left(x_{c}, y_{c}\right)+\pi .
$$

Another projection, as mentioned in Figure 10, on the plane formed with link 2 and link 3 can help to find the value of joint angle $2\left(\theta_{2}, 2\right)$ and joint angle $3\left(\theta_{3}, 3\right)$.

Law of cosines can be applied to obtain the joint angle 3 $\left(\theta_{3}, 3\right)$, as follows:

$$
\cos \theta_{3}=\frac{r^{2}+s^{2}-a_{2}^{2}-a_{3}^{2}}{2 a_{2} a_{3}} .
$$

By substituting the values of $r$ and $s$, these can be extracted by using Figure 8:

$$
\cos \theta_{3}=\frac{\left(x_{c}^{2}+y_{c}^{2}-d^{2}\right)+\left(z_{c}-d_{1}\right)^{2}-a_{2}^{2}-a_{3}^{2}}{2 a_{2} a_{3}} .
$$

As NM45 2.0 is inline, no shoulder is offset and, hence, $d=0$. 


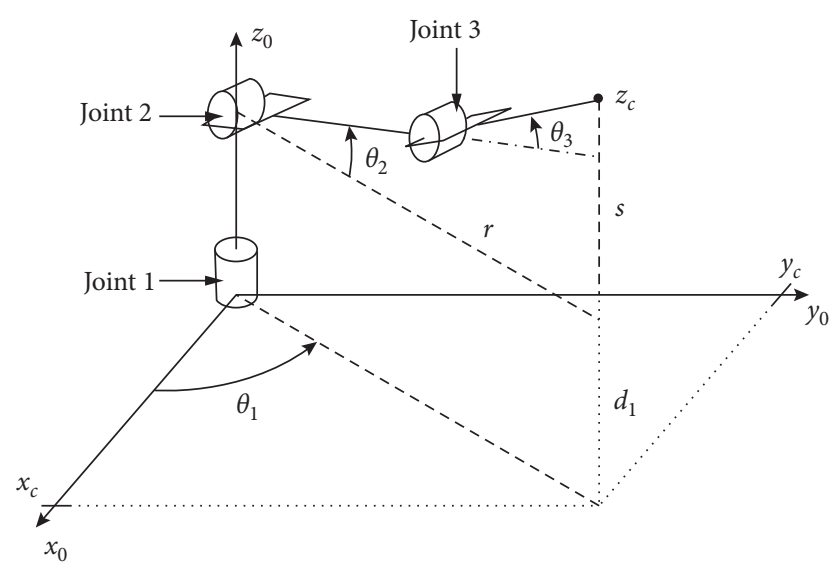

FIGURE 8: Comau NM45 (articulated arm) first three joints (Spong, Hutchinson, and Vidyasagar, 2006).

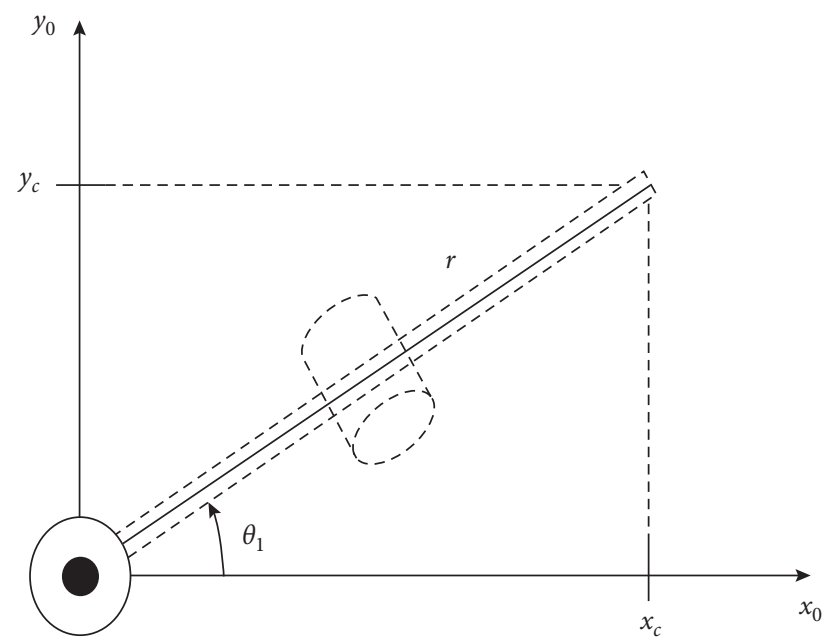

Figure 9: Projection of wrist centre onto $\mathrm{x} 0$, y0 plane (Spong, Hutchinson, and Vidyasagar, 2006).

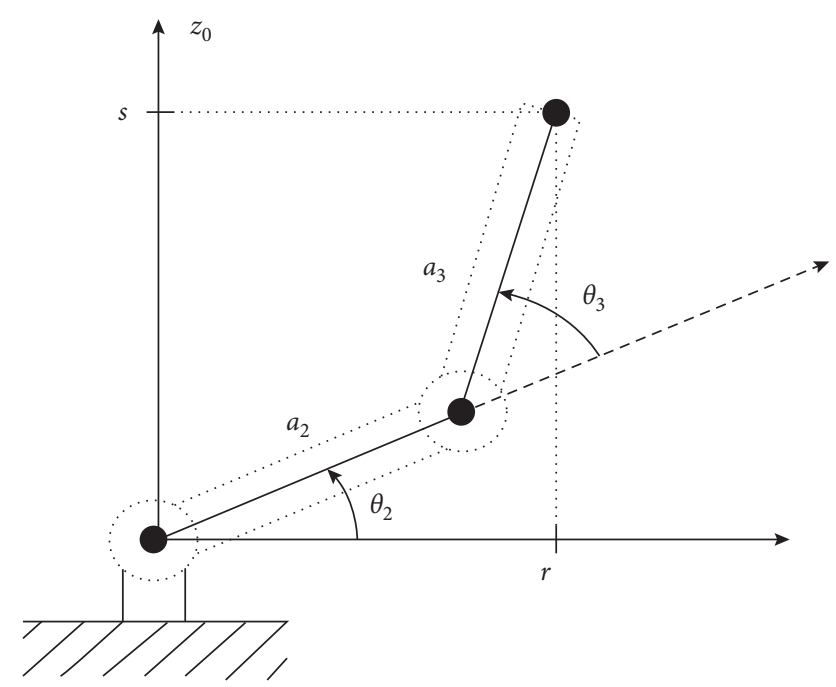

Figure 10: Projection on the plane (Link2 12, Link3 13) (Spong, Hutchinson, and Vidyasagar, 2006).
So,

$$
\cos \theta_{3}=\frac{x_{c}^{2}+y_{c}^{2}+\left(z_{c}-d_{1}\right)^{2}-a_{2}^{2}-a_{3}^{2}}{2 a_{2} a_{3}},
$$

where $a_{2}=L_{2}$ according to DH labelled figure, and

$$
\begin{aligned}
& \cos \theta_{3}=\frac{x_{c}^{2}+y_{c}^{2}+\left(z_{c}-d_{1}\right)^{2}-L_{2}^{2}-a_{3}^{2}}{2 L_{2} a_{3}}, \\
& \sin \theta_{3}=\sqrt{1-\cos \theta_{3}^{2}} .
\end{aligned}
$$

Also,

$$
\tan \theta_{3}=\sqrt{\frac{1-\cos \theta_{3}^{2}}{\cos \theta_{3}}}=\frac{\sqrt{1-D^{2}}}{D} .
$$

The value of $\theta_{3}$ can be written in terms of atan 2 and $D$ as follows:

$$
\theta_{3}=\operatorname{atan} 2\left(D, \pm \sqrt{1-D^{2}}\right)
$$

where + is for elbow up and - is for elbow down.

The projection in Figure 11 has been drawn onto the link 2 and link 3 plane to find $\theta_{2}$ :

$$
\begin{aligned}
& \theta_{2}=h 1-h 2, \\
& h 1=\mathrm{a} \tan 2(r, s)=\mathrm{a} \tan 2\left(\sqrt{x_{c}^{2}+y_{c}^{2}}, Z_{c}-d_{1}\right), \\
& h_{2}=\mathrm{a} \tan 2\left(a_{2}+a_{3} \cos \theta_{3}, a_{3} \sin \theta_{3}\right) .
\end{aligned}
$$

So, the value of $\theta_{2}$ can be written as follows:

$$
\begin{aligned}
\theta_{2}= & \operatorname{atan} 2\left(\sqrt{x_{c}^{2}+y_{c}^{2}, z_{c}-d_{1}}\right) \\
& -\operatorname{atan} 2\left(a_{2}+a_{3} \cos \theta 3, a 3 \sin \theta_{3}\right) .
\end{aligned}
$$

3.1. Finding Spherical Wrist Joint Values (Rotation) Using Euler Angles. The last three joint variables, $\theta 4, \theta 5, \theta 6$, are the Euler angles. So,

$$
\begin{aligned}
& \theta 4=\varnothing, \\
& \theta 5=\theta, \\
& \theta 6=\varphi .
\end{aligned}
$$

These angles are concerning the coordinate frame $o 3, x 3, y 3, z 3$. Now we need to calculate the transformation from $T_{4}^{3}$ to $T_{6}^{5}$ which is $T_{6}^{3}$ :

$$
T_{6}^{3}=\mathrm{A} 5 \mathrm{XA} 5 \mathrm{XA} 6=T_{4}^{3} \times T_{5}^{4} \mathrm{XT}_{6}^{5},
$$

where $T_{6}^{3}$ can be written in terms of $R_{6}^{3}$ and $O_{6}^{3}$ mentioned as follows:

$$
T_{6}^{3}=\left[\begin{array}{cc}
R_{6}^{3} & O_{6}^{3} \\
0 & 1
\end{array}\right]
$$

where $O_{6}^{3}$ is the position. 


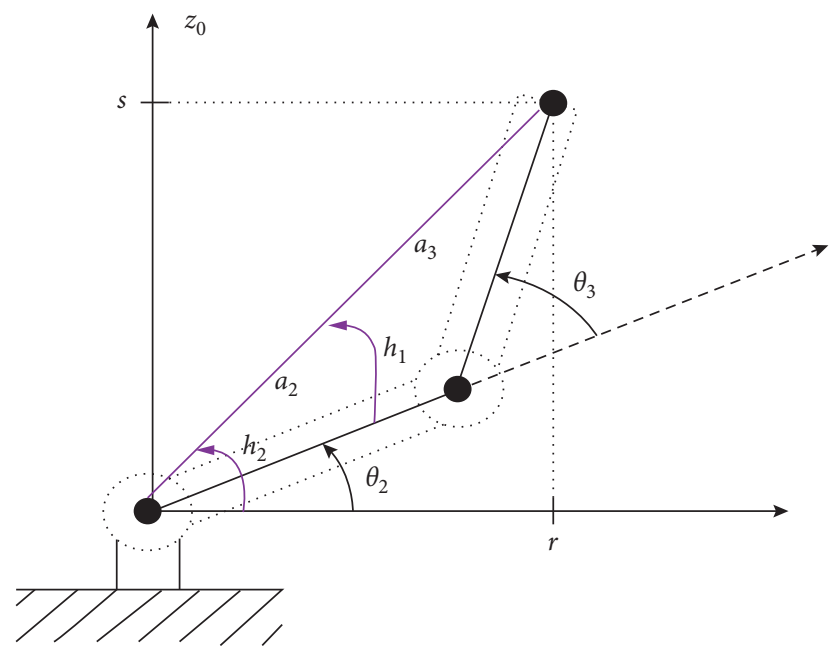

FIgURe 11: Projection onto 12 and 13 plane to find $\theta_{2}$. $\theta_{2}$ can be written in terms of angles $h 1$ and $h 2$.

For the spherical wrist, the ZYZ Euler transformation is needed. $\mathbf{R}_{6}^{3}$ is captured from equation $(22)$, so $\mathbf{R}_{6}^{3}$ can be written as

$$
\begin{aligned}
R_{\mathrm{ZYZ}} & =R_{6}^{3}=\left[\begin{array}{rrr}
\cos (\varnothing) \cos (\theta) \cos (\varphi)-\sin (\varnothing) \sin (\varphi) & -\cos (\varphi) \sin (\varnothing)-\cos (\varnothing) \cos (\theta) \sin (\varphi) & \cos (\varnothing) \sin (\theta) \\
\cos (\theta) \cos (\varphi) \sin (\varnothing)+\cos (\varnothing) \sin (\varphi) & \cos (\varnothing) \cos (\varphi)-\cos (\theta) \sin (\varnothing) \sin (\varphi) & \sin (\varnothing) \sin (\theta) \\
-\cos (\varphi) \sin (\theta) & \sin (\theta) \sin (\varphi) & \cos (\theta)
\end{array}\right], \\
R_{6}^{3} & =\left[\begin{array}{lll}
r 11 & r 12 & r 13 \\
r 21 & r 22 & r 23 \\
r 31 & r 32 & r 33
\end{array}\right]
\end{aligned}
$$

Whereas angles $a, b, m$ are $\varnothing, \theta, \varphi$, respectively.

The $\cos \theta$ can be extracted from $r 33$ from the following equation:

$$
\begin{aligned}
& \cos (b)=\cos \theta, \\
& \sin (b)=\sin \theta \sqrt{1-\cos ^{2} \theta} .
\end{aligned}
$$

The above $\cos \theta$ and $\sin \theta \operatorname{can}$ be written in form of $\tan$ to find the value of $\theta$ :

$$
\begin{aligned}
& \theta=\tan ^{-1}\left(\frac{\sqrt{1-\cos ^{2} \theta}}{r 33}\right), \\
& \theta=\tan ^{-1}\left(\frac{\sqrt{1-r 33^{2}}}{r 33}\right) .
\end{aligned}
$$

For nonsingular case, if both $r 13$ and $r 23$ are not zero, then equation (49) will yield the value of $\theta$ :

$$
\theta=a \tan 2\left(r 33, \pm \sqrt{1-r 33^{2}}\right),
$$

or

$$
\begin{aligned}
\tan \theta & =\frac{\sqrt{r 13^{2}+r 23^{2}}}{r 33}=\frac{\sqrt{(\cos (\varnothing) \sin (\theta))^{2}+(\sin (\varnothing) \sin (\theta))^{2}}}{\cos (\theta)}, \\
\theta & =\tan ^{-1}\left(\frac{\sqrt{r 13^{2}+r 23^{2}}}{r 33}\right) .
\end{aligned}
$$

Equation (51) shows the value of $\theta$ in terms of $r 13, r 23$ and $r 33$ :

$$
\theta=a \tan 2\left(r 33, \pm \sqrt{r 13^{2}+r 23^{2}}\right)
$$

The last column of $R_{6}^{3}$ from equation (46) can help to yield the value of $\varnothing$ :

$$
\tan \varnothing=\frac{r 23}{r 13}=\frac{\sin (\varnothing) \sin (\theta)}{\cos (\varnothing) \sin (\theta)}
$$

$\varnothing$ can be represented in terms of $r 13$ and $r 23$, as follows: 


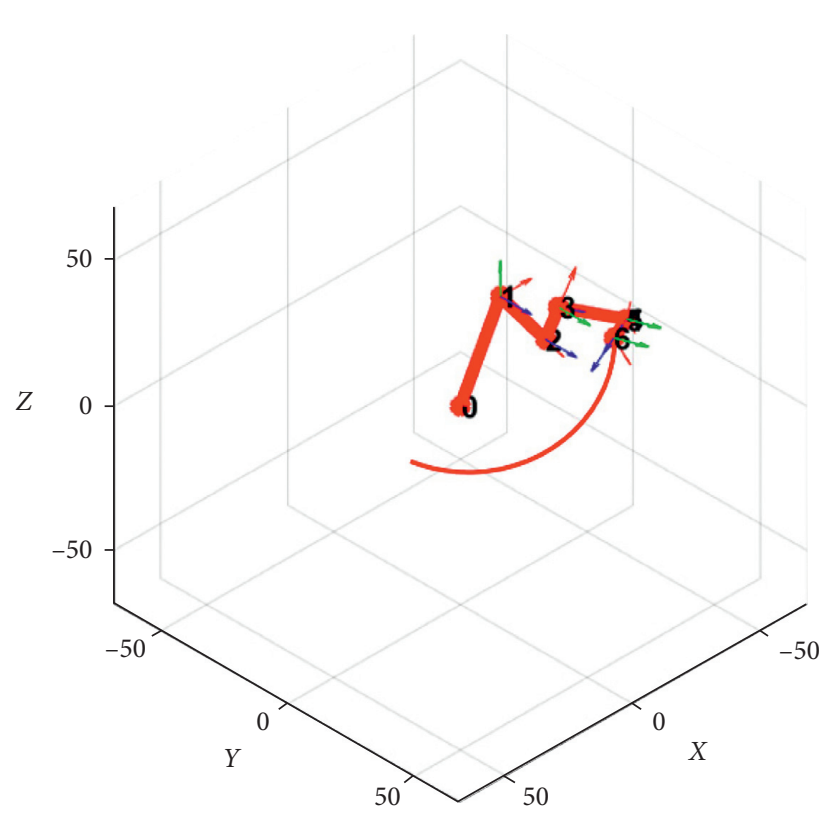

Figure 12: The trajectory of Comau NM45.

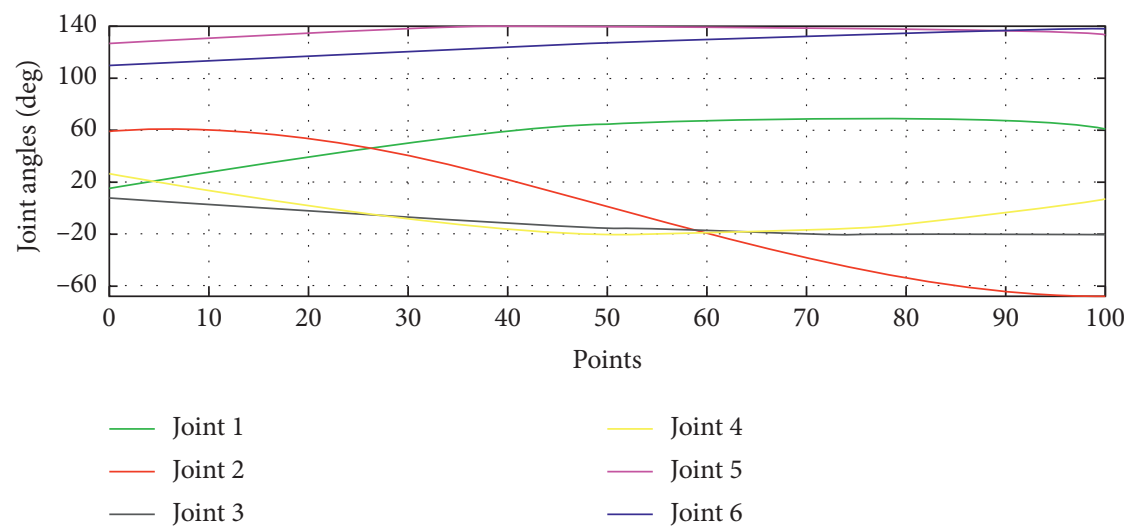

Figure 13: Joint trajectory of Comau NM45.

$$
\varnothing=a \tan 2(r 13, r 23) .
$$

For $\varphi$,

$$
\tan \varphi=\frac{r 32}{-r 21}=\frac{\sin (\theta) \sin (\varphi)}{-(-\cos (\varphi) \sin (\theta))}
$$

The $\varphi$ can be represented in terms of $r 31$ and $r 32$ mentioned as follows:

$$
\varphi=a \tan 2(-r 31, r 32) .
$$

For catering singularity, if $\sin (\theta)>0$, whereas $0<\theta<\pi$, then solution will be

$$
\begin{aligned}
& \varnothing=a \tan 2(-r 13,-r 23), \\
& \theta=a \tan 2\left(r 33, \pm \sqrt{r 13^{2}+r 23^{2}}\right), \\
& \varphi=a \tan 2(r 31,-r 32) .
\end{aligned}
$$

If $\sin (\theta)<0$, whereas $-\pi<\theta<0$, then the solution will be

$$
\begin{aligned}
& \varnothing=a \tan 2(-r 13,-r 23), \\
& \theta=a \tan 2\left(r 33, \pm \sqrt{r 13^{2}+r 23^{2}}\right), \\
& \varphi=a \tan 2(r 31,-r 32) .
\end{aligned}
$$

The last three joint values have been resolved by using the above equations of $\varnothing, \theta$, and $\varphi$.

For the trajectory planning, the program was written in Matlab by using forward and inverse kinematics equations mentioned in the above sections. The planned trajectory required the smooth motion of the end-effector. The joint angles were calculated using the inverse kinematics equations. Figure 12 shows the trajectory planning and robot motion along with the trajectory for the Comau NM45. The robot was able to follow the trajectory smoothly.

Figure 13 shows the mapping of the values of joints to move on 100 points to follow the trajectory. It shows that the achievement was smooth as there are no sudden spikes in the joint values. 


\section{Conclusion}

The modified DH convention has been used to perform the forward kinematics for the manipulator. The kinematics decoupling has been used to perform the inverse kinematics. The manipulator was divided into two parts to make the inverse kinematics problem simpler. The first 3 joints were resolved by using a geometrical approach, whereas the last three joints were resolved using the algebraic approach. The resultant kinematics solution was applied on the manipulator, and it was able to follow a test trajectory successfully. A similar approach can be used while solving the articulated robot with a spherical wrist. The techniques are applied for the $6-\mathrm{DoF}$ robot.

\section{Data Availability}

All the data used are presented as part of paper.

\section{Conflicts of Interest}

The authors declare that they have no conflicts of interest.

\section{References}

[1] Z. Gan and Q. Tang, "Robot kinematic calibration," in Visual Sensing and its Applications, pp. 166-192, Springer Berlin Heidelberg, Berlin, Germany, 2011.

[2] M. Ceccarelli, Fundamentals of Mechanics of Robotic Manipulationtle, Kluwer Academic Publishers, Dordrecht, Netherlands, 2004, http://books.google.co.uk/books?id=49eIUfSfOyg C\&printsec $=$ frontcover\&source $=$ gbs_ge_summary_r\&cad $=0 \#$ $\mathrm{v}=$ onepage $\& \mathrm{q} \& \mathrm{f}=$ false, 27 th edition.

[3] M. Shimizu, H. Kakuya, and W.-K. Yoon, "Analytical inverse kinematic computation for 7-DOF redundant manipulators with joint limits and its application to redundancy resolution," IEEE Transactions on Robotics, vol. 86, 2008.

[4] J. Denavit, "A kinematic notation for lower-pair mechanisms based on matrices," Journal of Applied Mechanics, pp. 215221, 1955.

[5] J. B Zhuang, “Review of drug treatment for Down's syndrome persons 'a complete and parametrically continuous kinematic model for robot manipulators', robotics and automation," American Journal of Mental Deficiency, vol. 80, no. 4, pp. 388-393, 1976.

[6] C. Robots, Coamu NM45 Specifications, Industrial-Robots, Robots, Bedford, MA, USA, 2015, http://industrial-robotics. co.uk/comau/nm_spec.htm.

[7] J. J. Craig, Introduction to Robotics: Mechanics and Control, Pearson Education, Inc. Pearson Prentice Hall, London, UK, pp. 1-388, 2005.

[8] M. Summers, "Robot capability test and development of industrial robot positioning system for the aerospace industry," SAE Technical Paper Series, vol. 114, no. 1, pp. 1108-1118, 2005.

[9] M.W. Spong, S. Hutchinson, and M. Vidyasagar, "Robot Modeling and Control," John Wiley \& Sons, Inc., Hoboken, NJ, USA, 2006.

[10] S. Megahed, "Inverse kinematics of spherical wrist robot arms: analysis and simulation," Journal of Intelligent and Robotic Systems, vol. 5, no. 3, pp. 211-227, 1992. 